\title{
Axial variation of wetting efficiency and liquid-solid mass transfer in long trickle bed columns
}

Elizabeth, L. du Toit, Rita Joubert, Francois Saayman, Willie Nicol*

Department of Chemical Engineering, University of Pretoria, South Africa

\begin{abstract}
The wetting efficiency and solid liquid mass transfer coefficient was measured separately and simultaneously using the electrochemical cell technique while varying the superficial liquid velocity and hydrodynamic state of the column by using different pre-wetting procedures. The wetting efficiency in columns with an aspect ratio - distance from distributor divided by column diameter - of up to 33, decreased with distance from the distributor regardless of the hydrodynamic state of the column. Although the solid liquid mass transfer coefficient also decreased with an increase in axial position, this decrease was much more significant where the pre-wetting procedure that resulted in a lower overall wetting efficiency was used. When a pre-wetting procedure that resulted in a higher average wetting efficiency was used, the liquid solid mass transfer coefficient was much less dependent on liquid velocity and only a relatively small decrease with axial position was observed. The results highlight the difference in surface renewal processes that is possible between columns operated at the same conditions but under different hydrodynamic states and emphasise the importance of hydrodynamic multiplicity when liquid redistribution is considered.
\end{abstract}

Keywords: Trickle bed reactor, Liquid-solid mass transfer, wetting efficiency, Electrochemical Measurement

\section{Introduction}

Trickle bed reactors are associated with low interaction flow regimes. External mass transport parameters and the extent of catalyst wetting are therefore amongst the most critical factors in terms of affecting the overall reaction rate. A wide variety of methods, including dissolution, electrochemical, reaction and absorption techniques, have been used for the quantification of liquid-solid mass transfer (LSMT) ${ }^{1,2}$. In all these studies, the effective LSMT coefficient, $\varphi k_{1 \mathrm{~s}}$, has always been measured without a separated measurement for wetting efficiency $(\varphi)$. The solid wetting efficiency is therefore intrinsically included in the determined value of the mass transfer coefficient. The prediction of the actual mass transfer coefficient at the solid surface in contact with the liquid subsequently requires the use of models for the 
quantification of the wetting efficiency $(\varphi)$ of the solid. These models predict a wide range of wetting efficiencies for the same operating conditions and it is very difficult to choose the most accurate one for a specific system ${ }^{3}$. This observation, in addition to the fact that the dependence of $\mathrm{k}_{\mathrm{ls}}$ and wetting efficiency on the hydrodynamic and physico-chemical parameters in the column may not necessarily be the same, makes this approach undesirable and an overall volumetric LSMT is often rather reported.

In addition to the abovementioned limitations, very few studies consider the spatial variation of the LSMT coefficient and a bed averaged value is generally reported. Where the spatial variation is considered, it is always as the combined parameter, $\varphi \mathrm{k}_{\mathrm{ls}_{\mathrm{s}}}$, Furthermore, all these studies use experimental set-ups with relatively low aspect ratios (bed length divided by column diameter) - typically less than 6.5. The aim of the work presented in this research note was to separately measure $\mathrm{k}_{\mathrm{ls}}$ and $\varphi$ as a function of axial position in trickle beds with a relatively high aspect ratio. For this purpose the measurement technique recently developed by Joubert and $\mathrm{Nicol}^{8}$ was used. This technique is adapted from the well-known electrochemical method for the measurement of LSMT and allows for simultaneous but separate measurement of $\mathrm{k}_{\mathrm{ls}}$ and $\varphi$ during the same experimental run and therefore under exactly the same hydrodynamic conditions. A lab scale column, which allowed for measurements up to an aspect ratio of 17 , as well as a pilot scale column, where measurements could be obtained at aspect ratios between 4.7 and 33, were used in this investigation. A variation in hydrodynamic state was obtained by employing different column pre-wetting techniques.

\section{Experimental}

Two separate clear PVC columns were used in this investigation. The first, with an internal diameter of $68 \mathrm{~mm}$, was $1.6 \mathrm{~m}$ long and the second larger column had an internal diameter of $104 \mathrm{~mm}$ and was $5 \mathrm{~m}$ long. Both columns were operated at ambient conditions - i.e. at approximately $298 \mathrm{~K}$ and with gas and liquid outlet at ca. $87 \mathrm{kPa}$. The liquid phase was fed through needle distributors, co-currently with the nitrogen gas phase. The superficial gas velocity was controlled at a relatively low value of $20 \mathrm{~mm} / \mathrm{s}$ by a Brooks Smart Mass Flow $5851 \mathrm{~S}$, while the superficial liquid phase velocities investigated were $3 \mathrm{~mm} / \mathrm{s}$ and $5 \mathrm{~mm} / \mathrm{s}$ as measured by an Electromagnetic Flow Measuring System (the PROline promag10H from Endress and Hauser). Hydrodynamic variation was achieved by using either the Kan- or Level pre-wetting procedure ${ }^{9}$. In the Kan pre-wetting mode, the liquid flow rate was 
increased until the pulsing regime was reached, after which the liquid flow rate was reduced and controlled at the desired set-point value. When the Levec pre-wetting procedure was followed, the bed was completely flooded by liquid and then drained for 30 minutes, after which the liquid and gas flow was introduced at a flow rate corresponding to the desired superficial velocity.

The electrolyte solution that was circulated through the columns, was an aqueous solution of $1 \mathrm{M} \mathrm{NaOH}, 0.02 \mathrm{M} \mathrm{K}_{4} \mathrm{Fe}(\mathrm{CN})_{6}, 0.003 \mathrm{M} \mathrm{K}_{3} \mathrm{Fe}(\mathrm{CN})_{6}$. The electrochemical reaction consisted of the reduction of Ferrycyanide on cathodes that consisted of multiple $4.5 \mathrm{~mm}$ diameter nickel coated bead clusters, distributed along the length of each column, and the reverse reaction at the anode - also a cluster of nickel coated particles at the bottom of each bed. At high enough voltages the rate of the electrochemical reaction is fast enough to ensure that mass transfer is rate controlling and the electrical current flowing in the solution between the electrodes is then a direct measure of the rate of mass transfer and can be calculated using equation 1:

$$
I=n C A_{\text {cathode }} \varphi k_{l s}
$$

The cathode area in this equation is based on a geometric calculation of the total area, while $\varphi$ is the fraction of the cathode that is actually in contact with the electrolyte - the cathode wetted fraction. Joubert and $\mathrm{Nicol}^{8}$ utilised the fact that the rate of the electrochemical reaction becomes the rate limiting step at very low voltages in order to quantify the surface area of the cathode that was exposed to the electrolyte solution. By varying the voltage during the same experimental run, it is subsequently possible to simultaneously measure the local LSMT coefficient and the wetting efficiency at each cathode. This allows one to calculate the specific local LSMT coefficient $\left(\mathrm{k}_{\mathrm{ls}}\right)$ based on the particle area that is actually in contact with the liquid phase. Joubert and $\mathrm{Nicol}^{8}$ determined that the electrochemical reaction is rate limiting at voltages below $50 \mathrm{mV}$ and subsequently a value of $20 \mathrm{mV}$ was used for the measurement of the wetting efficiency. A voltage of $1000 \mathrm{mV}$ was shown to lie in the centre of the mass transfer plateau and this value was used for all mass transfer measurements. The electrical current between a cathode and the anode under both applied voltage conditions, was measured with a NI PXI-4071 Digital Multi Meter with 7 1 2 digit accuracy. The cathode clusters were separated from each other by sections of $4 \mathrm{~mm}$ glass beads. 
a)

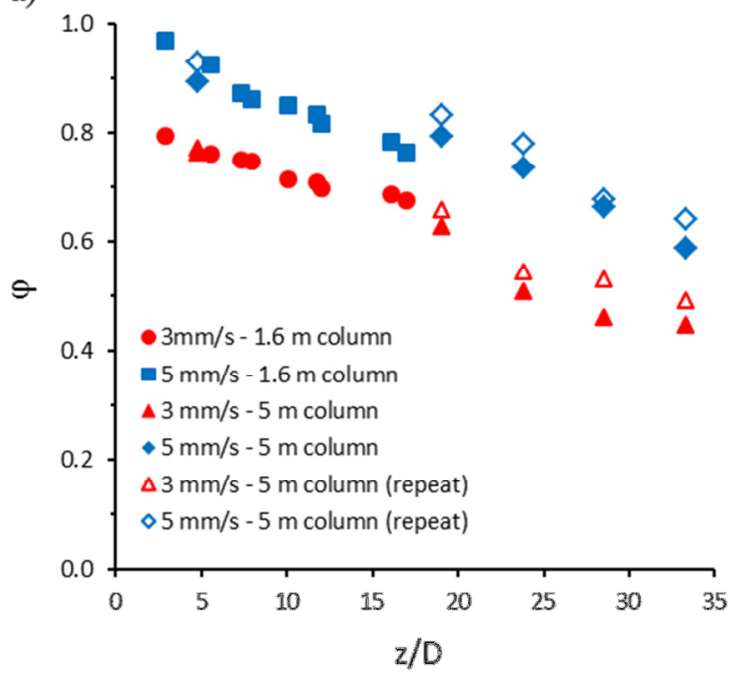

b)

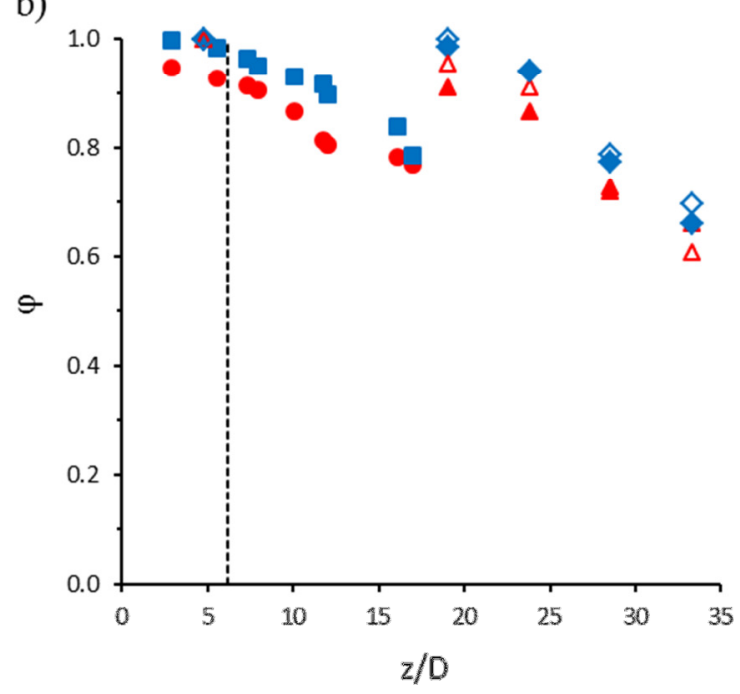

Figure 1. Wetting efficiency for a (a) Levec pre-wetted and (b) Kan pre-wetted bed as a function of aspect ratio. Superficial liquid velocities and column type indicated in the legend. Serrated line on b) shows the highest aspect ratio of previous studies where axial variation in wetting efficiency was considered.

\section{Results and Discussion}

Figure 1 shows the results of the wetting efficiency as a function of bed height - expressed as aspect ratio - for each of the two different pre-wetting modes. The reported results for the $1.6 \mathrm{~m}$ column are the averages of the 4 repeat runs - as the difference between all the measured variables between repeat runs were never more than $10 \%$. The results of both the repeat runs on the $5 \mathrm{~m}$ column are shown on the graphs. The Kan pre-wetted bed consistently resulted in more efficiently wetted particles throughout the length of the bed for both the velocities investigated. Furthermore, the wetting efficiency for the Levec pre-wetted bed monotonically decreases with bed depth. Although the wetting efficiency of the Kan prewetted bed also shows a decrease with increased axial position, there is a significant initial section of the bed where a stable value of wetting efficiency is observed. If the results of the two columns operated in this mode are considered separately, it also seems as if the axial position where the wetting efficiency starts to decrease is a function of the axial distance from the outlet of the bed and not the inlet. Baussaron et al. ${ }^{10}$ also observed a monotonic decrease in wetting efficiency with increased axial position on a bed of Alumina spheres where no pre-wetting occurred. However, they noticed no decline in wetting efficiency when the bed was properly pre-wetted. This discrepancy may be explained by the fact that the 
a)

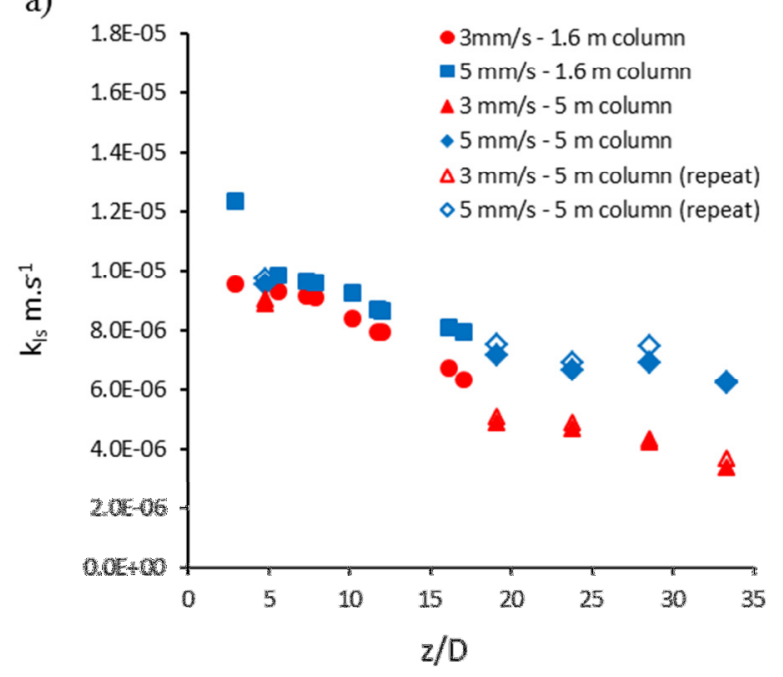

b)

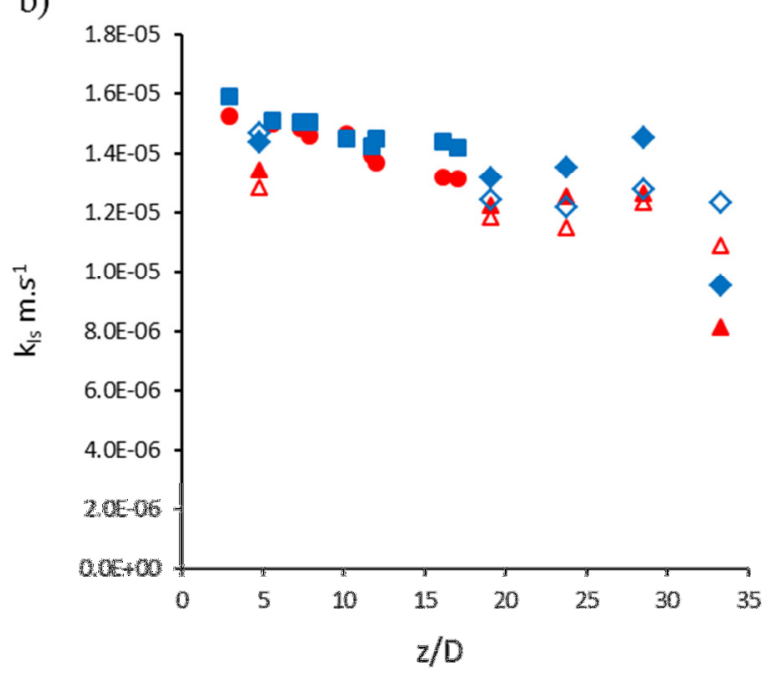

Figure 2. Liquid-solid mass transfer coefficient for a (a) Levec pre-wetted and (b) Kan prewetted bed as a function of aspect ratio. Superficial liquid velocities and column type indicated in the legend.

highest aspect ratio considered in their investigation was 6.14 (indicated by the solid line on figure $1(b)$ ) - which is still in the region where a constant value for wetting efficiency was observed in this investigation. Furthermore, the improved wettability of porous packing as compared to glass beads due to the liquid attracts liquid effect, as discussed by Schubert et $a l{ }^{11}$, may delay the axial position where a decrease in wetting efficiency becomes noticeable.

The LSMT coefficients calculated using the wetted fraction of the cathode beads are shown in figure 2. These values were calculated from equation 1 using the wetting efficiencies $(\varphi)$ given in figure 1 and the total cathode surface areas. Despite the fact that the local liquid solid mass transfer coefficient is now decoupled from particle wetting, a significant decrease in $\mathrm{k}_{\mathrm{ls}}$ is still observed during the Levec mode of operation. This is especially true at the lower superficial velocity investigated. If the quantitative decrease in $k_{1 s}$ is estimated by fitting a straight line through the data points at each velocity, a decrease of $66 \%$ is observed at 3 $\mathrm{mm} / \mathrm{s}$. At the higher superficial velocity investigated, the decline in $\mathrm{k}_{\mathrm{ls}}$ is much less significant (ca. 37\%). Contrary to these results, the $\mathrm{k}_{\mathrm{ls}}$ values in the Kan mode do not vary significantly with superficial velocity or bed height. On average, the $\mathrm{k}_{\mathrm{ls}}$ decreases by $16 \%$ if the data points of both velocities are considered. The observed $\mathrm{k}_{\mathrm{ls}}$ values in the Kan prewetting mode are also significantly higher than the values with Levec pre-wetting at corresponding superficial velocities. Loudon et al. ${ }^{9}$ showed that the liquid hold-up when the Kan pre-wetting procedure is used, is higher than that observed with the Levec pre-wetting procedure for all superficial velocities less than the flooding velocity. The interstitial 
velocities in the Kan-mode - calculated by dividing the superficial velocity by the dynamic liquid hold-up - is therefore consistently less than the values that will result in a bed operated under Levec pre-wetted conditions and can therefore not be used as indicator of the surface renewal processes for the hydrodynamic operating modes considered in this study. For the Levec pre-wetted bed the decline in $\varphi \mathrm{k}_{\mathrm{ls}}$ is a consequence of both a decrease in wetting efficiency combined with a reduction in the "quality" of flow where there is contact between the solid and the liquid. This supports the conclusions of Nicol and Joubert ${ }^{12}$, which confirmed the two-wetted zone-theory in trickle bed reactors and showed that more static liquid zones, where the rate of surface renewal processes are slow, exist in a Levec prewetted bed. In the Kan pre-wetted bed the decrease in the solid wetted fraction is the main contributing factor for an axial decline in $\varphi \mathrm{k}_{1 \mathrm{~s}}$. This suggests that most of the liquid in contact with the solid is in well irrigated zones in the bed, where reactant renewal is not a rate limiting process. This study highlights the difference in liquid flow morphology that must be present under different hydrodynamic states. Not only in terms of hydrodynamic parameters, but also in terms of surface renewal processes.

Finally, Schubert et al. ${ }^{11}$ points out that the hydrodynamic behaviour observed with glass beads, used in this study, as opposed to true porous catalysts are quite different due to the previously mentioned wetting boosting effect. However, the use of liquid redistribution in commercial reactors at a bed depth of $2-3 \mathrm{~m}$ as a rule of thumb ${ }^{13}$, may be related to the decrease in the effectiveness of the surface renewal processes with bed height observed in this work - either as a result of an increase in stagnant liquid zones or a decrease in the solid wetted fraction.

\section{Conclusions}

A new measurement technique, adapted from the electrochemical cell technique, made it possible to separately and simultaneously measure the axial variation of external wetting efficiency and the liquid solid mass transfer coefficient in relatively deep trickle bed columns. Under both the hydrodynamic states investigated, a significant decrease in wetting efficiency with distance from the distributor was observed. In the hydrodynamic state where the prewetting procedure that resulted in the higher end of the hydrodynamic spectrum was used, (Kan pre-wetting), the wetting efficiency remained constant for an initial section of the bed length, after which it continuously decreased. When the Levec pre-wetting procedure resulting in conditions at the lower end of the hydrodynamic envelope - was used, a 
monotonic decrease in wetting efficiency with axial position was observed. The liquid solid mass transfer coefficient in the Kan mode of operation was relatively insensitive to superficial liquid velocity. In addition only a small decrease in this parameter (ca. $16 \%$ on average) with axial position was observed under this hydrodynamic operating mode. A much more significant decrease in $\mathrm{k}_{\mathrm{ls}}$ resulted when the Levec pre-wetting procedure was followed. The extent of the decrease was strongly dependent on liquid superficial velocity with a $66 \%$ decrease with axial position at the lower superficial velocity investigated and $37 \%$ at the higher superficial velocity. The liquid solid mass transfer coefficient was significantly higher during the Kan-mode of operation over the entire range of aspect ratios considered for both liquid superficial velocities. This study highlights the difference in the surface renewal processes under different hydrodynamic modes of operation in trickle bed reactors- not only in terms of averaged values, but also in terms of location in the bed.

\section{Supporting Information}

More detail on the experimental section of this work is available free of charge via the Internet at http://pubs.acs.org/

\section{Nomenclature}

- Geometrically calculated electrode area $\left(\mathrm{m}^{2}\right)$

- Concentration $\left(\mathrm{mol} / \mathrm{m}^{3}\right)$

$k_{l s}$

- Current (A)

- Liquid solid mass transfer coefficient (m/s)

- Number of electrons involved in stoichiometric equation (-)

D - Column diameter (m)

$z \quad-$ Distance from distributor $(\mathrm{m})$

$\varphi \quad-$ Wetting efficiency (-)

\section{References}

(1) Dudukovic, M.P.; Larachi, F.; Mills, P.L. Multiphase reactors - Revisited. Chem. Eng.

Sci. 1999, 54, 1975.

(2) Dudukovic, M.P.; Larachi, F.; Mills, P.L. Multiphase catalytic reactors: A perspective on current knowledge and future trends. Catal. Rev. 2002, 44, 132. 
(3) Baussaron, L.; Julcour-Lebigue, C.; Boyer, C.; Wilhelm, A.M.; Delmas, H. Effect of partial wetting on liquid/solid mass transfer in trickle bed reactors. Chem. Eng. Sci. 2007, 62, 7020.

(4) Trivizadakis, M. E.; Karabelas, A. J. A study of local liquid/solid mass transfer in packed beds under trickling and induced pulsing flow. Chem. Eng. Sci. 2006, 61, 7684.

(5) Dang-Vu, T.; Doan, H.D.; Lohi, A.; Zhu, Y. A new liquid distribution factor and local mass transfer coefficient in a random packed bed. Chem. Eng. J. 2006, 123, 81.

(6) Gostick, J.; Doan, H. D.; Lohi, A.; Pritzker, M. D. Investigation of local mass transfer in a packed bed of pall rings using a limiting current technique. Ind. Eng. Chem. Res. 2003, 42, 3626.

(7) Joubert, R.; Nicol, W. Multiplicity behaviour of trickle flow liquid-solid mass transfer. Ind. Eng. Chem. Res. 2009, 48, 8387.

(8) Joubert, R.; Nicol, W. Trickle flow liquid-solid mass transfer and wetting efficiency in small diameter columns. Can. J. Chem. Eng. 2013, 91, 441.

(9) Loudon, D.; Van der Merwe, W.; Nicol, W. Multiple hydrodynamic states in trickle flow: Quantifying the extent of pressure drop, liquid holdup and gas-liquid mass transfer variation. Chem. Eng. Sci. 2006, 26, 7551.

(10) Baussaron, L.; Julcour-Lebigue, C.; Wilhelm, A.M.; Delmas, H.; Boyer, C. Wetting Topology in Trickle Bed Reactors. AIChE J. 2007, 53, 1850.

(11) Schubert, M.; Hessel, G.; Zippe, C.; Lange, R.; Hampel, U. Liquid flow texture analysis in trickle bed reactors using high-resolution gamma ray tomography. Chem. Eng. J. 2008, 140, 332.

(12) Nicol, W.; Joubert, R. Liquid-solid mass transfer distributions in trickle bed reactors. Chem. Eng. J. 2013, 230,361.

(13) Datsevich, L.B.; Dmitrii, A.M. Multiphase fixed-bed technologies; Comparative analysis of industrial processes (experience of development and industrial implementation). App. Cat. A: Gen. 2004, 261, 143. 